of the form $4 k+1$ or both of the form $4 k+3$, and is zero otherwise.

THEOREM 5. From $\mathrm{III}^{\prime}, N_{3}(n \equiv 6)=24 G^{\prime}(n)$.

Theorem 6. From $\mathrm{VI}^{\prime}, N_{3}(n \equiv 0)=8 G^{\prime}(n)+4 H(n)-4 e(n)$. $^{*}$

TheOREM 7. From $\mathrm{VI}^{\prime}, N_{3}(n \equiv 4)=8 G^{\prime}(n)+4 H(n)-8 e(n)$.*

Since $n \equiv 7(\bmod 8)$ cannot be represented as the sum of three squares, the set of formulas is complete.

It is clear that by selecting other functions $F(x, y, z)$ in a suitable manner other arithmetical results implicit in our general formulas may be obtained. As is usually the case in results of this type, strictly elementary proofs are no doubt possible, but are sometimes difficult to establish even after the theorems are known.

Creighton University

\title{
INVOLUTORY SYSTEMS OF CURVES ON RULED SURFACES $\dagger$
}

\section{A. F. CARPENTER}

In a paper presented to the International Mathematical Congress in Toronto in 1924, N. B. McLean discussed the properties of a certain one-parameter family of curves lying upon a ruled surface and characterized by the condition of forming a constant cross ratio with the complex curves of the surface. It is the purpose of the present paper to generalize McLean's system of curves and then to call attention to certain interesting special cases.

For the defining system of differential equations we make use of the form

(R) $y^{\prime \prime}+p_{12} z^{\prime}+q_{11} y+q_{12} z=0, \quad z^{\prime \prime}+p_{21} y^{\prime}+q_{21} y+q_{22} z=0$,

where $p_{12}^{\prime}=2 q_{12}, p_{21}^{\prime}=2 q_{21}$. For this form the two directrix curves $C_{y}, C_{z}$ are the two branches of the flecnode curve of $R$.

The tetrahedron of reference is that determined by the four points $P_{y}, P_{z}, P_{\rho}, P_{\sigma}$, where

$$
\rho=2 y^{\prime}+p_{12} z, \quad \sigma=2 z^{\prime}+p_{21} y,
$$

the unit point being so chosen that the general point of space will be represented by the expression

$$
x_{1} y+x_{2} z+x_{3} \rho+x_{4} \sigma .
$$

$\dagger$ Presented to the Society, April 16, 1938. 
The curves generated by the points $\eta=\alpha y+\beta z, \zeta=\alpha y-\beta z$, where $\alpha, \beta$ are arbitrary functions of the independent variable $x$, cut each generator $g$ of $R$ in points harmonically separated by the flecnode points of that generator. If the tangents to the four curves $C_{y}, C_{z}$, $C_{\eta}, C_{\zeta}$, at points in which they cut a generator of $R$ are to lie on a quadric, then the matrix of the coordinates of the general points on these tangents must be of rank two. The general points on these four tangent lines are given by the expressions

$$
\begin{aligned}
& y+2 \kappa y^{\prime}=y-p_{12} \kappa z+\kappa \rho, \quad z+2 \lambda z^{\prime}=-p_{21} \lambda y+z+\lambda \sigma, \\
& \eta+2 \mu \eta^{\prime}=\left(\alpha+2 \mu \alpha^{\prime}-p_{21} \beta \mu\right) y+\left(\beta+2 \mu \beta^{\prime}-p_{12} \alpha \mu\right) z+\alpha \mu \rho+\beta \mu \sigma, \\
& \zeta+2 \nu \zeta^{\prime}=\left(\alpha+2 \nu \alpha^{\prime}+p_{21} \beta \nu\right) y-\left(\beta+2 \nu \beta^{\prime}+p_{12} \alpha \nu\right) z+\alpha \nu \rho-\beta \nu \sigma,
\end{aligned}
$$

and the rank of the matrix of the coefficients of $y, z, \rho, \sigma$ in these expressions is two when

$$
\mu=\nu=\kappa \alpha /\left(\alpha-2 \kappa \alpha^{\prime}\right)=\lambda \beta /\left(\beta-2 \lambda \beta^{\prime}\right) .
$$

When conditions (2) are fulfilled the equation of the corresponding quadric is found to be

$$
x_{1} x_{4}-x_{2} x_{3}-p_{12} x_{3}^{2}+p_{21} x_{4}^{2}-2\left(\alpha^{\prime} / \alpha-\beta^{\prime} / \beta\right) x_{3} x_{4}=0 .
$$

If now $\alpha=c_{1} \theta_{1}(x), \beta=c_{2} \theta_{2}(x)$, where $\theta_{1}, \theta_{2}$ are arbitrary functions and $c_{1}, c_{2}$ constants, then

$$
\alpha^{\prime} / \alpha-\beta^{\prime} / \beta=\theta_{1}^{\prime} / \theta_{1}-\theta_{2}^{\prime} / \theta_{2},
$$

and it follows that there exists on $R$, for each choice of the ratio $\theta_{1} / \theta_{2} a$ one-parameter family of curves forming an involution such that the double elements are the two branches of the flecnode curve, and such that the tangents to the curves of each family at points of intersection with a generator of $R$ constitute one regulus of a quadric which is itself uniquely determined by the choice of $\theta_{1} / \theta_{2}$.

If $\theta_{1}=p_{21}{ }^{1 / 2}$ and $\theta_{2}=p_{12}{ }^{1 / 2}$, quadric (3) becomes

(4) $p_{12} p_{21}\left(x_{1} x_{4}-x_{2} x_{3}-p_{12} x_{3}^{2}+p_{21} x_{4}^{2}\right)+2\left(p_{12} q_{21}-p_{21} q_{12}\right) x_{3} x_{4}=0$,

the flecnode-complex quadric defined by McLean. The curves $C_{\eta}, C_{\zeta}$ are now generated by the points

$$
\eta=c_{1} p_{21}^{1 / 2} y+c_{2} p_{12}^{1 / 2} z, \quad \zeta=c_{1} p_{21}^{1 / 2} y-c_{2} p_{12}^{1 / 2} z .
$$

When $c_{1}=1, c_{2}=1, C_{\eta}$ and $C_{\zeta}$ are the complex curves of $R$. On the other hand, when $c_{1}=1, c_{2}=i$, the points determined by $\eta, \zeta$ are the involute points of $g$, and $C_{\eta}, C_{\xi}$ are the involute curves of $R$. McLean has shown that the quadric (4) may be determined by the generator $g$, 
the line of intersection of the planes osculating the two branches of the flecnode curve at their points of intersection with $g$, and the line of intersection of the planes osculating the two branches of the complex curve at their points of intersection with $g$. In view of the above facts, the theorem is equally true if for the complex curve we substitute the involute curve. Since for every generator on which the flecnode points are distinct either the complex points are real or the involute points are real, but not both pairs, the mystery of the existence of the quadric (4) when the complex points are imaginary is explained.

By definition all the quadrics given by (3) are tangent to $R$ along $g$. Since, no matter how $\alpha$ and $\beta$ are chosen, the points $P_{y}, P_{z}$ are double points of the involution on $g$, the tangents to $C_{y}, C_{z}$ at these points lie on each quadric. Thus all the quadrics have as a common intersection the generator $g$ of $R$ counted twice, together with the tangents at $P_{y}, P_{z}$ to the two branches of $R$ 's flecnode curve.

On each of the quadrics given by (3) lie, in general, two asymptotic tangents of $R$. That regulus of $R$ 's osculating quadric

$$
x_{1} x_{4}-x_{2} x_{3}=0
$$

whose lines intersect $g$ consists of asymptotic tangents of $R$. From (3) and (5) it is seen that the pair of planes

$$
p_{12} x_{3}^{2}+2\left(\alpha^{\prime} / \alpha-\beta^{\prime} / \beta\right) x_{3} x_{4}-p_{21} x_{4}^{2}=0
$$

contains the complete intersection of (3) and (5). These planes intersect in $g$ and are tangent planes to (5), their points of tangency being given by the expressions

$$
\left\{\beta^{\prime} / \beta-\alpha^{\prime} / \alpha \pm\left[\left(\beta^{\prime} / \beta-\alpha^{\prime} / \alpha\right)^{2}+p_{12} p_{21}\right]^{1 / 2}\right\} y+p_{12} z .
$$

Asymptotic tangents to $R$ at these points thus lie on the quadrics given by (3).

If we suppose in particular that $\alpha^{\prime} / \alpha-\beta^{\prime} / \beta=0$, so that $\alpha / \beta=c_{1} / c_{2}$ $=$ const., then (7) becomes, after division by $\pm p_{12}{ }^{1 / 2}$,

$$
p_{21}^{1 / 2} y \pm p_{12}^{1 / 2} z \text {. }
$$

But these are the expressions for the complex points of $g{ }^{*}$ There is thus seen to exist on $R$ a one-parameter involutory family of curves whose double curves are the two branches of the flecnode curve and which are such that any four curves of the family cut all generators of $R$ in constant cross ratio. The tangents to these curves along any generator con-

* Wilczynski, Projective Differential Geometry, p. 207. 
stitute one regulus of a quadric whose equation may be written in the form

$$
x_{1} x_{4}-x_{2} x_{3}-p_{12} x_{3}^{2}+p_{21} x_{4}^{2}=0,
$$

and on this quadric lie the asymptotic tangents of $R$ at the complex points of $g$. That regulus of this quadric which consists of the tangents to the $\eta, \zeta$ curves is generated by the two axial pencils

$$
x_{3}-\kappa x_{4}=0, \quad x_{1}-\kappa\left(x_{2}+p_{12} x_{3}\right)+p_{21} x_{4}=0,
$$

whose axes are, respectively, the generator $g$ and the line joining $P_{y^{\prime}}$ to $P_{z^{\prime}}$. The other regulus is generated by the two axial pencils

$$
x_{2}+p_{12} x_{3}-\lambda x_{4}=0, \quad x_{1}-\lambda x_{3}+p_{21} x_{4}=0,
$$

whose axes are, respectively, the tangents to the two branches of the flecnode curve at $P_{y}, P_{z}$.

Without going into further detail we may state that when $\eta=c_{1} y+c_{2} z, \zeta=c_{1} y-c_{2} z$ the lines of intersection of the osculating planes of pairs of curves $C_{\eta}, C_{\zeta}$, at points $P_{\eta}, P_{\zeta}$ constitute one regulus of a quadric whose equation in the reference system here employed, where $p=p_{12} q_{21}-p_{21} q_{12}, q=q_{11}-q_{22}$, is

$$
\begin{aligned}
p\left(x_{1} x_{4}-x_{2} x_{3}\right. & \left.-p_{12} x_{3}^{2}+p_{21} x_{4}^{2}\right) \\
& -2 q\left(p_{12} x_{1} x_{3}-p_{21} x_{2} x_{4}-q_{12} x_{3}^{2}-q x_{3} x_{4}+q_{21} x_{4}^{2}\right)=0 .
\end{aligned}
$$

This quadric also is seen to be tangent to $R$ along $g$ and to have in common with (5) and (8) the two asymptotic tangents at the complex points of $g$.

Let us next assume that $\alpha^{\prime} / \alpha-\beta^{\prime} / \beta=c$, and hence that $\alpha / \beta=a e^{c x}$. Equation (3) now becomes

$$
x_{1} x_{4}-x_{2} x_{3}-p_{12} x_{3}^{2}+p_{21} x_{4}^{2}-2 c x_{3} x_{4}=0,
$$

which appears to define a one-parameter family of quadrics. But a transformation $\xi=c x$ of the independent variable, which has no geometric significance, effects the following transformations in the coefficients of system $(R)$ and in the reference system:

$p_{i k}=c \bar{p}_{i k}, \quad q_{i k}=c^{2} \bar{q}_{i k}, \quad x_{1}=\bar{x}_{1}, \quad x_{2}=\bar{x}_{2}, \quad c x_{3}=\bar{x}_{3}, \quad c x_{4}=\bar{x}_{4}$.

It follows that the constant $c$ drops out of equation (9) and we have

$$
x_{1} x_{4}-x_{2} x_{3}-p_{12} x_{3}^{2}+p_{21} x_{4}^{2}-2 x_{3} x_{4}=0 ;
$$

so no generality is lost by taking $c=1$.

If we write $u=a e^{x}$ so that $\eta=u^{1 / 2} y+u^{-1 / 2} z, \zeta=u^{1 / 2} y-u^{-1 / 2} z$, the system of differential equations in $\eta$ and $\zeta$ becomes 


$$
\begin{aligned}
\eta^{\prime \prime} & +\frac{1}{2}\left(p_{12} u+p_{21} u^{-1}\right) \eta^{\prime}-\frac{1}{2}\left(p_{12} u-p_{21} u^{-1}+2\right) \zeta^{\prime} \\
& +\frac{1}{4}\left(p_{12} u-p_{21} u^{-1}+2 q_{12} u+2 q_{21} u^{-1}+2 q_{11}+2 q_{22}+1\right) \eta \\
& -\frac{1}{4}\left(p_{12} u+p_{21} u^{-1}+2 q_{12} u-2 q_{21} u^{-1}-2 q\right) \zeta=0 \\
\zeta^{\prime \prime} & +\frac{1}{2}\left(p_{12} u-p_{21} u^{-1}-2\right) \eta^{\prime}-\frac{1}{2}\left(p_{12} u+p_{21} u^{-1}\right) \zeta^{\prime} \\
& +\frac{1}{4}\left(p_{12} u+p_{21} u^{-1}+2 q_{12} u-2 q_{21} u^{-1}+2 q\right) \eta \\
& -\frac{1}{4}\left(p_{12} u-p_{21} u^{-1}+2 q_{12} u+2 q_{21} u^{-1}-2 q_{11}-2 q_{22}-1\right) \zeta=0 .
\end{aligned}
$$

Now while on each quadric given by (3), and hence on quadric (10), there lie two asymptotic tangents of $R$, it does not follow that there will be two asymptotic curves of $R$ included in each one-parameter family. If two asymptotic curves of a ruled surface are chosen for directrix curves, the defining system of differential equations is characterized by the absence of certain terms of the first order.* In order that the two curves given by (11) be asymptotic curves it would be necessary for both the coefficient of $\zeta^{\prime}$ in the first and that of $\eta^{\prime}$ in the second to vanish; but this is impossible. If either vanishes without the other, one of the curves is asymptotic. A more interesting situation develops when we impose a slightly greater restriction on the coefficients in (11). We shall first assume that the coefficients of both $\eta^{\prime}$ and $\zeta^{\prime}$ in the first equation of (11) vanish, that is

$$
p_{12} u+p_{21} u^{-1}=0, \quad p_{12} u-p_{21} u^{-1}+2=0 .
$$

This makes $C_{\eta}$ an asymptotic curve of $R$.

From (12), since $u=a e^{x}, p_{12}^{\prime}=2 q_{12}, p_{21}^{\prime}=2 q_{21}$, we find by differentiation that

$$
\begin{aligned}
& p_{12} u-p_{21} u^{-1}+2 q_{12} u+2 q_{21} u^{-1}=0, \\
& p_{12} u+p_{21} u^{-1}+2 q_{12} u-2 q_{21} u^{-1}=0 .
\end{aligned}
$$

Making use of (12) and (13), we may now write system (11) as

$$
\begin{gathered}
\eta^{\prime \prime}+\frac{1}{4}\left(2 q_{11}+2 q_{22}+1\right) \eta+\frac{1}{2} q \zeta=0, \\
\zeta^{\prime \prime}-2 \eta^{\prime}+\frac{1}{2} q \eta+\frac{1}{4}\left(2 q_{11}+2 q_{22}+1\right) \zeta=0 .
\end{gathered}
$$

Equations (12) place certain restrictions on the coefficients of system (R). We find that $a p_{12}=-e^{-x}, p_{21}=a e^{x}$ and hence $2 a q_{12}=e^{-x}, 2 q_{21}=a e^{x}$. Assumptions (12) have thus particularized four of the six coefficients of $(\mathrm{R})$ and have at the same time introduced an arbitrary constant $a$. But this is not an essential constant, for the transformation $z=a \bar{z}$, which has no geometric significance, removes the $a$ and leaves system $(\mathrm{R})$ in the form

* Wilczynski, op. cit., p. 142. 


$$
y^{\prime \prime}-e^{-x} z^{\prime}+q_{11} y+\frac{1}{2} e^{-x} z=0, \quad z^{\prime \prime}+e^{x} y^{\prime}+\frac{1}{2} e^{x} y+q_{22} z=0 .
$$

If now in (11) we replace $p_{12}, p_{21}, 2 q_{12}, 2 q_{21}$ with the respective values $-e^{-x}, e^{x}, e^{-x}, e^{x}$, we obtain the system of differential equations satisfied by $\eta, \zeta$ in the form

$$
\begin{aligned}
& \eta^{\prime \prime}-\frac{1}{2}(a-1 / a) \eta^{\prime}+\frac{1}{2}(a+1 / a-2) \zeta^{\prime}+\frac{1}{4}\left(2 q_{11}+2 q_{22}+1\right) \eta+\frac{1}{2} q \zeta=0, \\
& \zeta^{\prime \prime}-\frac{1}{2}(a+1 / a+2) \eta^{\prime}+\frac{1}{2}(a-1 / a) \zeta^{\prime}+\frac{1}{2} q \eta+\frac{1}{4}\left(2 q_{11}+2 q_{22}+1\right) \zeta=0,
\end{aligned}
$$

and when $a=1$, (16) reduces to (14). It may be verified that the directrix curves employed in system (14) constitute the two branches of the involute curve of that special type of surface $R$ defined by the flecnode system (15).

If, proceeding as above, we equate to zero the coefficients of $\eta^{\prime}$ and $\zeta^{\prime}$ in the second equation of (11), system (11) reduces to

$$
\begin{aligned}
\eta^{\prime \prime}-2 \zeta^{\prime}+\frac{1}{4}\left(2 q_{11}+2 q_{22}+1\right) \eta+\frac{1}{2} q \zeta & =0, \\
\zeta^{\prime \prime}+\frac{1}{2} q \eta+\frac{1}{4}\left(2 q_{11}+2 q_{22}+1\right) \zeta & =0,
\end{aligned}
$$

so that $C_{\boldsymbol{\zeta}}$ is now an asymptotic curve. At the same time system (R) reduces to a form which is identical with (15) after use has been made of the transformation $z=-a \bar{z}$. Equation (17) is recognized as that special case of (16) for which $a=-1$.

That the exponential coefficients of (15) do not occur in (16) is not surprising when it is seen that the transformation $z=e^{x} \bar{z}$, which leaves the directrix curves unchanged, reduces (15) to the form

$$
y^{\prime \prime}-\bar{z}^{\prime}+q_{11} y-\frac{1}{2} \bar{z}=0, \bar{z}^{\prime \prime}+y^{\prime}+2 \bar{z}^{\prime}+\frac{1}{2} y+\left(q_{22}+1\right) \bar{z}=0,
$$

in which the only variable coefficients are $q_{11}$ and $q_{22}$.

For system (15) we find that

$$
\rho=2 y^{\prime}-e^{-x_{z}}, \quad \sigma=2 z^{\prime}+e^{x} y .
$$

By successive differentiation and the use of (15) and (19) we get

$$
\begin{aligned}
\rho^{\prime \prime}-e^{-x} \sigma^{\prime}+q_{11} \rho+\frac{1}{2} e^{-x} \sigma & =-2 q_{11}^{\prime} y+\frac{1}{2}\left(2 q_{11}+2 q_{22}+1\right) e^{-x} z \\
\sigma^{\prime \prime}+e^{x} \rho^{\prime}+\frac{1}{2} e^{x} \rho+q_{22} \sigma & =-\frac{1}{2}\left(2 q_{11}+2 q_{22}+1\right) e^{x} y-2 q_{22}^{\prime} z .
\end{aligned}
$$

If now we impose upon system (15) the further conditions that $q_{11}$ and $q_{22}$ are unequal constants but are such that

$$
2 q_{11}+2 q_{22}+1=0
$$

then (20) discloses the fact that the curves $C_{\rho}, C_{\sigma}$ are the two branches of the flecnode curve of a ruled surface projectively equivalent to $R$. Wilczynski named the surface generated by the line $l_{\rho \sigma}$ the derivative surface of $R$. 
The two sheets $F_{1}, F_{2}$ of $R$ 's flecnode surface are generated by the flecnode tangents $l_{y \rho}$ and $l_{z \sigma}$. On the first sheet $C_{y}$ figures as one branch of its flecnode curve, and on the second sheet $C_{z}$ plays the same role. The points given by the expressions $\theta=-e^{x} y, \phi=\rho+y$ are the flecnode points of $l_{y \rho}$. By differentiation, making use of (15), (19), and (21), we find that

$$
\phi^{\prime \prime}-e^{-x} \theta^{\prime}+q_{11} \phi+\frac{1}{2} e^{-x} \theta=0, \quad \theta^{\prime \prime}+e^{x} \phi^{\prime}+\frac{1}{2} e^{x} \phi+q_{22} \theta=0 .
$$

The points given by the expressions $\xi=e^{-x} z, \psi=\sigma-z$, are the flecnode points on $l_{z \sigma}$. Proceeding as above we find that

$$
\xi^{\prime \prime}-e^{-x} \psi^{\prime}+q_{11} \xi+\frac{1}{2} e^{-x} \psi=0, \quad \psi^{\prime \prime}+e^{x} \xi^{\prime}+\frac{1}{2} e^{x} \xi+q_{22} \psi=0 .
$$

Because of the identity of forms of (15), (22), and (23), it follows that for the particular type of ruled surface which is defined by (15) and for which

$$
q_{11}=c_{1} \neq c_{2}=q_{22}, \quad 2 q_{11}+2 q_{22}+1=0,
$$

the two sheets of its flecnode surface are projectively equivalent to it.

The assumptions on $q_{11}$ and $q_{22}$ reduce (16) to the form

$$
\begin{aligned}
& \eta^{\prime \prime}-\frac{1}{2}(a-1 / a) \eta^{\prime}+\frac{1}{2}(a+1 / a-2) \zeta^{\prime}+\frac{1}{2} q \zeta=0, \\
& \zeta^{\prime \prime}-\frac{1}{2}(a+1 / a+2) \eta^{\prime}+\frac{1}{2}(a-1 / a) \zeta^{\prime}+\frac{1}{2} q \eta=0 ;
\end{aligned}
$$

and from (24) by successive differentiation and elimination we obtain the fourth order differential equations defining the curves $C_{\eta}, C_{\zeta}$ of the one-parameter family defined by (24). These equations are found to be identical in form and free from the arbitrary constant $a$, that for $C_{\eta}$ being $\eta^{(4)}+q \eta^{\prime}-\frac{1}{4} q^{2} \eta=0$. It follows that the curves of this family are all projectively equivalent, and since $q=$ const., they are anharmonic curves.*

To sum up, we have demonstrated the existence of a one-parameter family of ruled surfaces defined by a system of differential equations

$$
\begin{gathered}
y^{\prime \prime}-e^{-x} z^{\prime}+q_{11} y+\frac{1}{2} e^{-x} z=0, \quad z^{\prime \prime}+e^{x} y^{\prime}+\frac{1}{2} e^{x} y+q_{22} z=0, \\
q_{11}=c_{1} \neq c_{2}=q_{22}, \quad 2 q_{11}+2 q_{22}+1=0,
\end{gathered}
$$

for each of which the derivative surface as well as each sheet of the flecnode surface is projectively equivalent to the given surface, and on which there lies a one-parameter involution family of curves whose double elements are the two branches of the flecnode curve, all curves of the family being projectively equivalent anharmonic curves.

The University of Washington

* Wilczynski, op. cit., p. 279. 